\title{
Methicillin-Resistant Staphylococcus Aureus Nasal Carriers among Laboratory Technical Staff of Tertiary Hospital in
} Eastern Nepal

\author{
Neupane R, Bhatt N, Poudyal A, Sharma A
}

\author{
Corresponding Author \\ Navin Bhatt \\ Department of Microbiology \\ BP Koirala Institute of Health Sciences, \\ Dharan, Nepal. \\ E-mail: n.navin.bhatt@gmail.com
}

\section{Citation}

Neupane R, Bhatt N, Poudyal A, Sharma A. Methicillin-Resistant Staphylococcus Aureus Nasal Carriers among Laboratory Technical Staff of Tertiary Hospital in Eastern Nepal. Kathmandu Univ Med J. 2020;69(1):3-8.

\begin{abstract}
Background

Methicillin-resistant Staphylococcus aureus (MRSA) has been the leading cause of nosocomial and community infections worldwide. In particular, healthcare workers are at constant risk to develop MRSA carriage. There is a paucity of data regarding the epidemiology of MRSA in laboratory workers who are constantly in contact with these pathogens in almost every hospital.
\end{abstract}

\section{Objective}

This study was undertaken to identify the burden of MRSA nasal carriers and the antibiotic susceptibility pattern of the isolates among laboratory technical staff.

\section{Method}

Sterile nasal swabs were taken from 50 laboratory technical staff working in the Central and Emergency laboratory of BP Koirala Institute of Health Sciences (BPKIHS). Standard procedures were followed for isolation, identification and antibiotic sensitivity testing. Antibiotic susceptibility tests were performed using disc diffusion and growth on Oxacillin screen agar based on Clinical and Laboratory Standards Institute (CLSI) guidelines.

\section{Result}

Out of 50 isolates of Staphylococcus aureus, 44 (88\%) were Methicillin-sensitive (MSSA) while six (12\%) were Methicillin-resistant (MRSA). All 50 isolates of S. aureus were found resistant to Penicillin while all were found sensitive to Teicoplanin, Linezolid, and Levofloxacin. Only five (10\%) were sensitive to Erythromycin while 49 (98\%) were sensitive to both Amikacin and Gentamicin. However, none of the MRSA isolates were found sensitive to all tested antimicrobial agents.

\section{Conclusion}

This repertoire portrays the emergence of MRSA in laboratory workers which clearly indicates the necessity of complying with the sanitary and antibacterial guidelines and adhering to the rational use of antibiotics to prevent nosocomial infections.

\section{KEY WORDS}

Antibiotic resistance, Laboratory personnel, MRSA, Staphylococcus aureus 


\section{INTRODUCTION}

Staphylococcus aureus is a major human pathogen that causes a variety of illnesses ranging from minor skin infections to major life-threatening diseases. ${ }^{1,2}$ In clinical practice, $S$. aureus has been a global problem due to the emergence of antibiotic-resistant strains, and one amongst many is Methicillin-Resistant Staphylococcus aureus (MRSA). MRSA is considered to be one of the leading pathogens implicated in severe hospital-acquired infections. Nasal carriage of MRSA plays an important role in the epidemiology and pathogenesis of infection and is becoming an increasing problem among healthcare workers (HCWs) and in the healthy community individuals., Besides, due to the limitation on the therapeutic choices, the treatment for infections caused by MRSA has become a serious health problem. ${ }^{3}$ In particular, HCWs are at constant risk to develop MRSA carriage. They may serve as an agent of cross-contamination and are thus an important carrier between hospitals and communities in nosocomial infections. ${ }^{5,6}$

In Nepal, the overall rate of nasal carriage of MRSA among HCWs is $7.5 \%$ and it is found to be highest among the laboratory personnel (10.5\%).4 HCWs are the major source of hospital-acquired MRSA transmission in developing countries like Nepal. ${ }^{5}$ A known history of MRSA infection or colonization, direct contact with patients with MRSA infections, recent hospitalization or emergency department visits, and recent antibiotic use are some of the common risk factors for MRSA carriage in $\mathrm{HCWs}^{7}$ In addition, the development of resistance or acquisition of MRSA is most established with the administration of multiple antibiotics. ${ }^{8,9}$

Screening and eradication of MRSA via infection control programs targeting the HCWs in the hospital have been commended as an important step in the prevention of MRSA infection. , $^{4}$ Furthermore, continuous surveillance on the antibiotic resistance profiles of local $S$. aureus isolates and formulating antibiotic policies is found to be effective to prevent therapeutic crisis due to MRSA infections. ${ }^{2}$ This study was undertaken to identify the burden of MRSA nasal carriers and the antibiotic susceptibility pattern of the isolates among the laboratory technical staff working in our hospital.

\section{METHODS}

This was a cross-sectional study conducted among 50 laboratory technical staff working at Central and Emergency laboratory at BPKIHS for a duration of 4 months from March to June 2018. Central laboratory consists of Biochemistry, Microbiology, Hematology, and Serology units whereas the Emergency laboratory is a single combined laboratory. Prior to the conduction of the study, ethical approval was taken from the Department Research Unit (DRU), an authorized research review wing of the Institutional
Review Committee (IRC) of BPKIHS (Reference number: 962/Micro/075). Similarly, informed written consent was obtained from every patient after insuring them the confidentiality of the data.

Laboratory technical staff who provided consent to participate in the study, who had no signs of infections nor any other systemic diseases and hadn't been under antibiotics or nasal medications were included in the study. However, laboratory personnel who were suffering from upper respiratory tract infections or any other systemic diseases, having immunocompromised state due to any cause, using any nasal medications, undertaking antibiotics or had taken antibiotics within the last 1 month were excluded from the study.

Sample Size: Using the sample size for finite population,

Sample size $(\mathrm{n})=\mathrm{N}\left(4 p q / \mathbb{R}^{2}\right) /\left\{\left(4 p q / \mathbb{R}^{2}\right)+\mathrm{N}-1\right\}$

Taking prevalence of MRSA ${ }^{10}$

(p) $=15.4$, complement of $p(q)=84.6$,

Allowable error $(I)=5 \%$ of $p$ Thus, $n=49.71$,

Thus, we took the sample size as 50 .

Nasal swabs were collected from the anterior nares of the participants by using sterile cotton swabs. The swab sticks were rotated for about 4-5 times inside each anterior nares and were immediately transported to the Microbiology laboratory for further processing. Isolation, identification, and determination were done based on the standard microbiological technique. Following all aseptic precautions, the samples were inoculated onto blood agar and MacConkey agar plates and were incubated overnight at $37 .{ }^{11}$ The confirmed growth was identified by using different biochemical tests and antimicrobial susceptibility testing was further performed on Mueller Hinton Agar (MHA) by Kirby Bauer's disc diffusion method recommended by Clinical and Laboratory Standards Institute (CLSI) guidelines. ${ }^{12}$

\section{Identification $^{13}$}

Suspected smooth, densely opaque colonies sometimes surrounded by a narrow zone of hemolysis with yellow pigment on blood agar plate, corresponding minute pink, lactose fermenting colonies on MacConkey agar plate were further processed. Identification of Staphylococcus aureus was done on colony morphology, gram stain, catalase test, and coagulase test.

\section{Catalase test ${ }^{13}$}

Catalase test was performed. The rapid production of bubbles (effervescence) was interpreted as a positive test.

\section{Slide coagulase test ${ }^{11}$}

This test is used for the detection of clumping factor, present in Staphylococcus aureus and absent from most other Staphylococci. The reagent is human plasma. Citrated 
human plasma was used and it was stored at $4^{\circ} \mathrm{C}$ for daily use, brought it to room temperature before use.

Suspected Staphylococcal colony was emulsified in a drop of saline on a microscope slide with a minimum spreading, making a smooth and milky suspension. Similarly, the suspension of positive control (S. aureus ATCC 25923) and negative control ( $E$. coli ATCC 25922) strains were made to confirm proper reactivity of the plasma. The sterile straight inoculating wire loop was dipped into the undiluted plasma at the room temperature and was stirred into the staphylococcal suspension on the slide. Then, the wire was flamed and the same process was repeated for the control suspensions.

Coarse clumping of cocci visible within 10 seconds was read as positive. An absence of clumping or any reaction taking more than 10 seconds to develop was considered as negative.

Test Staphylococcal suspension was compared with both negative and positive suspensions strains.

\section{Tube Coagulase Test ${ }^{11}$}

One in six dilutions of plasma was prepared in saline $(0.85 \%$ $\mathrm{NaCl}$ ) and from that diluted plasma $1 \mathrm{ml}$ was placed in small tubes. The tubes were numbered as-

\section{For positive control strain}

2. For negative control strain

\section{For test organism}

\section{For uninoculated plasma}

A suspected staphylococcal colony was emulsified in test tube 3 of diluted plasma. Similarly, with each batch of tests, tubes with positive control and negative control were emulsified in $1 \mathrm{ml}$ volume of diluted plasma in positive and negative marked tubes. Once, the tube of unseeded diluted plasma was placed to confirm that it does not clot spontaneously.

The tubes were inoculated at $37^{\circ} \mathrm{C}$, up to four hours. The tubes were examined in one, two and four hours to see for any clot formation by tilting the tubes through 900 and were left at room temperature overnight. The next day, conservation of plasma into the still gel (coagulum) that remained in place when the tube was tilted and clots floating in the fluid was observed as positive. The tube in which the plasma remained wholly liquid or only a flocculent or ropy precipitate was a negative test. The test tube was compared to the positive and negative control tubes.

\section{Disc Diffusion testing ${ }^{12}$}

Antimicrobial disc diffusion testing was performed on $\mathrm{MHA}$ plates and interpreted according to CLSI guidelines. $S$. aureus ATCC 25922 was used as a control and tested daily along with test strains.

\section{Inoculum preparation}

With the help of straight inoculating wire, 3-5 wellisolated colonies of the same morphology were picked and transferred into a tube containing $5 \mathrm{ml}$ of peptone water broth. The broth culture was incubated at 35 until the turbidity of $0.5 \mathrm{McF}$ arland standard was achieved. The comparison was done visually in the adequate light against a card with a white background and contrasting black lines.

\section{Inoculation of test plates ${ }^{12}$}

After the achievement of turbidity, a sterile cotton swab was dipped into inoculum suspension within 15 minutes. Any excess inoculum from the swab was removed by rotating and firmly pressing it on the inside wall of the tube above the fluid level. The swab was streaked over the entire surface of the dried sterile MHA plate. This procedure was repeated twice by rotating and streaking the plates at approximately 600 each time to ensure the even distribution of inoculum. The rim of agar was swabbed in the final step.

\section{Application of disks to inoculated agar plates ${ }^{12}$}

Antimicrobial disks supplied by HiMedia Laboratories, India impregnated with Penicillin (10units), Erythromycin $(15 \mu \mathrm{g})$, Teicoplanin $(30 \mu \mathrm{g})$, Linezolid $(30 \mu \mathrm{g})$, Gentamicin $(10 \mu \mathrm{g})$, Levofloxacin $(5 \mu \mathrm{g})$ and Amikacin $(30 \mu \mathrm{g})$ antibiotics were used.

These disks were dispensed onto the surface of the inoculated agar plate. Each disk was pressed down to ensure complete contact with the agar surface. The disk from their center was kept at least $24 \mathrm{~mm}$ apart. No more than 6 disks were placed on $90 \mathrm{~mm}$ plates. The plates were inverted and incubated at 35 \pm 2 for $16-18$ hours after the application of disks.

\section{Reading plates and interpreting results ${ }^{12}$}

Each plate was examined after 16-18 hours of incubation. Zones of complete inhibition including the diameter of the disk were read by the unaided eye. The nearest whole millimeter was noted as the zone with the help of a ruler, held on the back of an inverted Petri plate. The reading of the plate was done a few inches above a black, nonreflecting background, illuminated with reflected light. No obvious, visible growth area was considered as zone margin. Any faint growth of tiny colonies detected only with a magnifying lens at the edge of the zone of inhibited growth, was ignored. However, sub-culture was made for the discrete colonies growing within a clear zone of inhibition. The size of the zone of inhibition was interpreted according to CLSI guidelines and the organisms were reported as susceptible, intermediate or resistant to particular agents accordingly.

\section{Interpretation of Antimicrobial susceptibility ${ }^{12}$}

Oxacillin screening agar Base (for detection of MRSA) 
Oxacillin screening agar Base (for detection of MRSA)

\begin{tabular}{|llll|}
$\begin{array}{l}\text { Concentrations of } \\
\text { antibiotics }\end{array}$ & \multicolumn{3}{c|}{ Zone of inhibition (diameter in mm) } \\
\hline & Susceptible & Intermediate & Resistant \\
\hline Penicillin (10 units) & $\geq 29$ & - & $\leq 28$ \\
\hline Erythromycin $(15 \mu \mathrm{g})$ & $\geq 23$ & $14-22$ & $\leq 13$ \\
\hline Teicoplanin $(30 \mu \mathrm{g})$ & $\geq 14$ & $11-13$ & $\leq 10$ \\
\hline Linezolid(30 $\mu \mathrm{g})$ & $\geq 21$ & - & $\leq 20$ \\
\hline Gentamicin $(30 \mu \mathrm{g})$ & $\geq 18$ & $16-17$ & $\leq 16$ \\
\hline Levofloxacin(5 $\mu \mathrm{g})$ & $\geq 19$ & $16-18$ & $\leq 15$ \\
\hline Amikacin $(30 \mu \mathrm{g})$ & $\geq 18$ & $16-17$ & $\leq 16$ \\
\hline
\end{tabular}

\section{Preparation of Media ${ }^{14}$}

Thirty-five grams of MHA (HiMedia) powder was poured into $1000 \mathrm{ml}$ of distilled water in a clean sterile conical flask. It was mixed properly and 40 grams of $\mathrm{NaCl}$ was added to it (i.e. $4 \%$ of $\mathrm{NaCl}$ ). After thorough mixing, it was autoclaved at $1210 \mathrm{C}$ and $15 \mathrm{lbs}$ for 30 minutes. 0.006 grams $(6 \mu \mathrm{g} / \mathrm{ml})$ of antibiotic Oxacillin powder was added to the agar. The media was mixed gently and carefully in order to obtain a homogenous distribution of the antibiotic and $20 \mathrm{ml}$ volume poured carefully into the sterile Petri plate to avoid air bubbles. The plates were then set on a flat horizontal surface and allowed to solidify undisturbed for at least 10 minutes. Two control plates, prepared under the same condition but without antibiotics was included in every screening test.

\section{Reading of test Result ${ }^{14}$}

The growth on the spotted area was carefully examined in transmitted light. Any visible, hazy growth or $>1$ colony was recorded as resistant.

Data were entered in Microsoft Excel 2013 (Microsoft Corporation, Redmond, Washington, USA) and statistical analysis was done using SPSS 17.0 version (Chicago, Inc). Descriptive statistics were used to explore the characteristics of data by calculating percentage, mean and range. Chi-square test was used to compare the categorical variables. Appropriate graphs and bar diagrams were prepared for graphical representation.

\section{RESULTS}

A total of 50 laboratory workers were screened from various laboratory departments for MRSA, among them 35(70\%) were males and $15(30 \%)$ were females. Majority of the participants, i.e. $21(42 \%)$ belonged to the age group of 30 39 years followed by $20(40 \%)$ to $40-49$ years and $9(18 \%)$ to the age group of 20-29 years, respectively. In this study, all nasal swabs were obtained from various departments of Central and Emergency laboratories. Majority, i.e. 20(40\%) were from Emergency laboratory followed by $14(28 \%)$ from Microbiology, 9(18\%) from Hematology, 5(10\%) from Biochemistry and 2(4\%) from Serology units of central laboratory, respectively. (Table 1)
Table 1. Demographic characteristics of the participants

\begin{tabular}{|lcc|}
\hline Variables & $\begin{array}{c}\text { No. of } \\
\text { participants }\end{array}$ & Percentage (\%) \\
\hline Gender & 35 & 70 \\
\hline Male & 15 & 30 \\
\hline Female & & \\
\hline Age groups & 9 & 18 \\
\hline $20-29$ & 21 & 42 \\
\hline $30-39$ & 20 & 40 \\
\hline $40-49$ & & \\
\hline Department & 20 & 40 \\
\hline Emergency & 14 & 28 \\
\hline Microbiology & 2 & 4 \\
\hline Serology & 5 & 10 \\
\hline Biochemistry & 9 & 18 \\
\hline Hematology & & \\
\hline
\end{tabular}

Among the 50 samples that were all isolated as $S$. aureus when treated in Oxacillin screening agar, six (12\%) isolates were Oxacillin resistant (MRSA), while 44 (88\%) isolates were Oxacillin sensitive (MSSA).

Meanwhile, out of six (12\%) MRSA isolates, an equal number of MRSA i.e., three (15\%) were from Emergency and three $(21.42 \%)$ were from the Microbiology department. No MRSA was detected from Biochemistry, Hematology and Serology departments. Categorizing demographically, four (67\%) of the MRSA positive isolates were males whereas rest $(33 \%)$ were females. Besides, participants with age group 40-49 had the highest number (50\%) of MRSA positive isolates, followed by participants of age group 3039 years (33\%) and $20-29$ years (17\%) respectively. (Table 2 )

Table 2. Sensitivity pattern of the isolates according to their demographic characteristics

\begin{tabular}{|c|c|c|c|c|c|}
\hline \multirow[t]{2}{*}{ Variables } & \multirow[t]{2}{*}{$\begin{array}{c}\text { No. of } \\
\text { participants }\end{array}$} & \multicolumn{2}{|c|}{$\begin{array}{l}\text { Sensitivity } \\
\text { Pattern }\end{array}$} & \multirow[b]{2}{*}{ Chi-square } & \multirow[b]{2}{*}{$p$ value } \\
\hline & & MSSA & MRSA & & \\
\hline \multicolumn{6}{|l|}{ Gender } \\
\hline Male & 35 & 31 & 4 & 0.036 & 0.849 \\
\hline Female & 15 & 13 & 2 & & \\
\hline \multicolumn{6}{|l|}{ Age groups } \\
\hline $20-29$ & 9 & 9 & 1 & 0.418 & 0.811 \\
\hline $30-39$ & 21 & 1.9 & 2 & & \\
\hline $40-49$ & 20 & 16 & 3 & & \\
\hline
\end{tabular}

\section{Antibiotic susceptibility patterns}

No difference was seen in the resistance pattern between MSSA and MRSA. All (100\%) the isolates of Staphylococcus aureus were susceptible to Teicoplanin, Linezolid, and Levofloxacin. Susceptibility to Amikacin and Gentamicin was noted to be equal at $98 \%$ and to Erythromycin was 
$10 \%$. At the same time, all the isolates were found to be penicillin-resistant. (Table 3)

Table 3. Antibiotic Susceptibility Pattern

\begin{tabular}{|llllll|}
\hline Antibiotics & \multicolumn{1}{c}{$\begin{array}{c}\text { No. of } \\
\text { participants }\end{array}$} & \multicolumn{2}{c}{ MSSA } & MRSA & \\
\hline Penicillin & 50 & $\begin{array}{l}\text { Sensi- } \\
\text { tive }\end{array}$ & $\begin{array}{l}\text { Resis- } \\
\text { tant }\end{array}$ & $\begin{array}{l}\text { Sensi- } \\
\text { tive }\end{array}$ & $\begin{array}{l}\text { Resis- } \\
\text { tant }\end{array}$ \\
\hline Teicoplanin & 50 & 0 & $100 \%$ & 0 & $100 \%$ \\
\hline Linezolid & 50 & $100 \%$ & 0 & $100 \%$ & 0 \\
\hline Erythromycin & 50 & $100 \%$ & 0 & $100 \%$ & 0 \\
\hline Amikacin & 50 & $10 \%$ & $90 \%$ & $10 \%$ & $90 \%$ \\
\hline Levofloxacin & 50 & $98 \%$ & $2 \%$ & $98 \%$ & $2 \%$ \\
\hline Gentamicin & 50 & $100 \%$ & 0 & $100 \%$ & 0 \\
\hline
\end{tabular}

\section{DISCUSSION}

This study was done to assess the distribution of Methicillin-Resistant Staphylococcus aureus (MRSA) nasal carrier among laboratory technical staff in the tertiary hospital in Eastern Nepal. A total of 50 nasal swabs were included in this study which was taken and processed in the Microbiology laboratory.

MRSA carriage rate among healthcare workers was found to be higher in this study indicating the possibility of hospitalacquired MRSA colonization and may be due to the lack of personal protective gear in the hospital setting.

In our study, $12 \%$ of the isolates were found to be Methicillin resistant (MRSA). A similar study conducted by Rongpharpi et al. in 2013 in India, reported that the Methicillin resistance was seen in $11.43 \%$ among health care workers, and in the study done by Shibabaw et al. in 2013 in Ethiopia, Methicillin resistance was seen to be $12.7 \%$ which also corresponds to this study findings. ${ }^{15,16}$ However, a study conducted by Kaminsky et al. in Germany showed the nasal MRSA prevalence among health care workers to be $5.3 \%$ and in another study in Argentina by Boncompain et al. the prevalence rate of MRSA was $6.3 \%$ which are lower compared to our study. ${ }^{17,18}$ Additionally, separate studies from Nepal and Pakistan reported that among the healthcare workers, laboratory personnel had the highest carriage rate of MRSA which was $10.5 \%$ and $25 \%$ respectively. ${ }^{4,6}$

The gender differentiation of the isolates showed that $35(70 \%)$ were taken from the male participants and 15 (30\%) were taken from the female participants. Among them, $4(11.42 \%)$ out of 35 males and $2(13.33 \%)$ out of 15 females tested positive for MRSA. Similar was the result of an Ethiopian study where more females (14.1\%) were colonized by MRSA than males (6.2\%). ${ }^{19}$

Among the Departments, the scenario in the Emergency Department was poorer than others with the overall positivity in MRSA cases being reported at 15\% (3 out of 20). The worst case was that of the Microbiology department of the Central laboratory which reported $21.42 \%$ (3 out of 14) to be MRSA positive. No MRSA was detected in Hematology, Serology and Biochemistry departments. The high prevalence of MRSA in the microbiology lab may be due to direct exposure to the pathogens during the processing time as well as the general vicinity of the infected samples to the laboratory personnel. The high rate of MRSA in the Emergency Department can also be attributed to the fact that all samples are streamlined in the Emergency Department, hence increasing the chances of infection as well as increased level of resistance.

All 50 isolates of $S$. aureus were resistant to Penicillin while all were sensitive to Teicoplanin, Linezolid, and Levofloxacin. Similarly, $98 \%$ were sensitive to both Amikacin and Gentamicin, while only $10 \%$ were sensitive to Erythromycin. Comparable to our study, maximum resistance $(100 \%)$ to penicillin was reported in a study by Tahnkiwale et al. ${ }^{20}$ Similarly, various other studies also reported the sensitivity of Linezolid to nasal $S$ aureus as $100 \% .{ }^{15,21}$ However according to Pavani Gandham et al, the reported resistance to Linezolid is about $2-20 \%$ which is higher resistance compared to our study. ${ }^{22}$

Staphylococcus aureus often has increased resistance to macrolides. In our study, $90 \%$ of isolates were resistant to Erythromycin. This finding is in agreement with the result of the study conducted in western Nepal (94.5\%) by Subedi et al. ${ }^{23}$

In comparison to other studies, our study showed that the $S$. aureus isolates had increased susceptibility to Gentamicin. The resistance of $2 \%$ was noted in our study. Whereas study by Farzana et al. showed resistance of $58 \%$ to Gentamycin. ${ }^{24}$ Increased trend of resistance was noticed in Canada (25.9\%), USA (35.5\%), Latin America (91.2\%), Europe $(71.7 \%)$ and western Pacific region (74.0\%) which was higher than our study results. ${ }^{25}$

In the present study, none of the MRSA isolates included were found to be sensitive to all of the antimicrobial agents tested. This was in accordance with the study reported by Subedi et al which found $100 \%$ MRSA resistance to more than one drug. ${ }^{23}$

Due to the lack of other antibiotic discs in the laboratory, determination of susceptibility could not be done which could have highlighted the susceptibility of specific antibiotics against MRSA in our setting. Moreover, this is a hospital-based cross-sectional study conducted in a small group of laboratory technical staff. A larger study including other health care workers specifically doctors, nurses, operation theatre staff would lead to results with increased applicability and impact. 


\section{CONCLUSION}

This report depicts the emergence of MRSA in the laboratory population indicating that MRSA is not only limited to infected patients of the hospital, but also among the healthcare workers. Moreover, the detection of MRSA with multidrug resistance has added the complexity of the problem.

\section{REFERENCES}

1. Masalha M, Borovok I, Schreiber R, Aharonowitz Y, Cohen G. Analysis of Transcription of the Staphylococcus aureus Aerobic Class $\mathrm{lb}$ and Anaerobic Class III Ribonucleotide Reductase Genes in Response to Oxygen. Journal of bacteriology. 2001 Dec15;183(24):7260-72.

2. Solberg CO. Spread of Staphylococcus aureus in hospitals: causes and prevention. Scandinavian journal of infectious diseases. 2000 Jan 1;32(6):587-95.

3. Mahmoud AM, Albadawy HS, Bolis SM, Bilal NE, Ahmed AO, Ibrahim ME. Inducible clindamycin resistance and nasal carriage rates of Staphylococcus aureus among healthcare workers and community members. African health sciences. 2015;15(3):861-7.

4. Khatri S, Pant ND, Bhandari R, Shrestha KL, Shrestha CD, Adhikari $\mathrm{N}$, et al. Nasal carriage rate of methicillin resistant staphylococcus aureus among health care workers at a tertiary care hospital in kathmandu, nepal. Journal of Nepal Health Research Council. 2017 Aug 13;15(1):26-30.

5. Khanal $R$, Sah $P$, Lamichhane $P$, Lamsal A, Upadhaya S, Pahwa VK. Nasal carriage of methicillin resistant Staphylococcus aureus among health care workers at a tertiary care hospital in Western Nepal. Antimicrobial resistance and infection control. 2015 Dec;4(1):39.

6. Salman MK, Ashraf MS, Iftikhar S, Baig MA. Frequency of nasal carriage of Staphylococcus Aureus among health care workers at a Tertiary Care Hospital. Pakistan journal of medical sciences. 2018 Sep;34(5):1181.

7. Schubert M, Kämpf D, Jatzwauk L, Kynast F, Stein A, Strasser R, Dulon $M$, Nienhaus A, Seidler A. Prevalence and predictors of MRSA carriage among employees in a non-outbreak setting: A cross-sectional study in an acute care hospital. Journal of Occupational Medicine and Toxicology. 2019 Dec;14(1):7.

8. Tiwari HK, Das AK, Sapkota D, Sivrajan K, Pahwa VK. Methicillin resistant Staphylococcus aureus: prevalence and antibiogram in a tertiary care hospital in western Nepal. The journal of infection in developing countries. 2009 Oct 22;3(09):681-4.

9. Kluytmans J, Van Belkum A, Verbrugh H. Nasal carriage of Staphylococcus aureus: epidemiology, underlying mechanisms, and associated risks. Clinical microbiology reviews. 1997 Jul1;10(3):50520.

10. Vinodhkumaradithyaa A, Uma A, Shirivasan M, Ananthalakshmi I, Nallasivam $P$, Thirumalaikolundusubramanian $P$. Nasal carriage of methicillin-resistant Staphylococcus aureus among surgical unit staff. Jpn J Infect Dis. 2009 May 1;62(3):228-9.

11. D. Baird, "Staphylococcus: Cluster Forming Gram Positive Cocci," In: J. G. Collee, A. G. Fraser, B. P. Marmion and A. Simmons, Eds., Mackie and McCartney Practical Medical Microbiology. 1996; 2: 245-61.

12. CLSI guideline 2017, M100S

13. Winn, Washington C, and Elmer W. Koneman. Gram positive cocci. In: Koneman's Color Atlas and Textbook of Diagnostic Microbiology. Philadelphia: Lippincott Williams \& Wilkins, 2006.
To prevent the spreading of resistant carriers of $S$. aureus, the first step is the compliance of laboratory personnel with the sanitary and antibacterial guidelines. Healthcare workers should be periodically educated and trained about hygiene maintenance and infection control and the effects of the rampant use of antibiotics. Furthermore, implementation of the screening programs and infection control measures as well as treatment of the MRSA positive isolates are of utmost importance to prevent the spread of MRSA carriage among healthcare workers.

14. Murray, Patrick R., and Ellen Jo Baron. Manual of Clinical Microbiology. Washington, D.C.: ASM Press, 2003.

15. Sharon Rainy Rongpharpi, Naba Kumar Hazarika, and Hitesh Kalita, The Prevalence of Nasal Carriage of Staphylococcus Aureus Among Healthcare Workers at a Tertiary Care Hospital in Assam with Special Reference to MRSA. J Clin Diagn Res. 2013 Feb; 7(2): 257-60.

16. Shibabaw A, Abebe T, Mihret A. Nasal carriage rate of methicillin resistant Staphylococcus aureus among Dessie Referral Hospital health care workers; Dessie, Northeast Ethiopia. Antimicrobial resistance and infection control. 2013 Dec;2(1):25.

17. Kaminski A, Kammler J, Wick M, Muhr G, Kutscha-Lissberg F. Transmission of methicillin-resistant Staphylococcus aureus among hospital staff in a German trauma centre: a problem without a current solution? Bone \& Joint Journal. 2007 May1; 89(5):642-5.

18. Boncompain CA, Suárez CA, Morbidoni HR. Staphylococcus aureus nasal carriage in health care workers: First report from a major public hospital in Argentina. Revista Argentina de microbiologia. $2017 \mathrm{Apr}$ 1;49(2):125-31.

19. Gebreyesus A, Gebre-Selassie S, Mihert A. Nasal and hand carriage rate of methicillin resistant Staphylococcus aureus (MRSA) among health care workers in Mekelle Hospital, North Ethiopia. Ethiopian medical journal. 2013 Jan;51(1):41-7.

20. Tahnkiwale SS, Roy S, Jalgaonkar SV. Methicillin resistance among isolates of Staphylococcus aureus: antibiotic sensitivity pattern \& phage typing. Indian journal of medical sciences. 2002 Jul;56(7): 330-4.

21. Raut S, Bajracharya K, Adhikari J, Pant SS, Adhikari B. Prevalence of methicillin resistant Staphylococcus aureus in Lumbini Medical College and Teaching Hospital, Palpa, Western Nepal. BMC research notes. 2017 Dec;10(1):187.

22. Gandham P. Linezolid resistant Staphylococcus aureus. International Journal of Research in Medical Sciences. 2017 Jan 26;2(4):1253-6.

23. Subedi S, Brahmadathan KN. Antimicrobial susceptibility patterns of clinical isolates of Staphylococcus aureus in Nepal. Clinical microbiology and infection. 2005 Mar 1;11(3):235-7.

24. Farzana K, Hameed A. Resistance pattern of clinical isolates of Staphylococcus aureus against five groups of antibiotics. Journal of Research (Science). 2006;17(1):19-26.

25. Diekema DJ, Pfaller MA, Schmitz FJ, Smayevsky J, Bell J, Jones RN, Beach, M. and SENTRY Participants Group, 2001. Survey of infections due to Staphylococcus species: frequency of occurrence and antimicrobial susceptibility of isolates collected in the United States, Canada, Latin America, Europe, and the Western Pacific region for the SENTRY Antimicrobial Surveillance Program, 1997-1999. Clinical Infectious Diseases, 32(Supplement_2), pp.S114-S132. 